\title{
Linkage of Azurophil Granule Secretion in Neutrophils to Chloride Ion Transport and Endosomal Transcytosis
}

\author{
Claus Fittschen and Peter M. Henson \\ Department of Pediatrics, National Jewish Center for Immunology and Respiratory Medicine, Denver, Colorado 80206
}

\begin{abstract}
Neutrophils contain at least two types of secretory granules. The present work links the secretion of the (lysosomal type) azurophil granules, but not that of specific granules, to endosomal transport mechanisms. (a) Selective stimulation of azurophil granule secretion by the Na-ionophore Monensin, or nonselective stimulation by FMLP after cytochalasin B pretreatment elicited marked pinocytic activity in parallel with azurophil granule release, whereas FMLP alone, selective for specific granules, elicited little fluid pinocytosis. ( $b$ ) Pinosomes thus formed fused with azurophil granules, suggesting that exocytosis of azurophil granules might occur via endosomal organelles.

This hypothesis was tested by determining the effect on the endosomal pathway (s) of two treatments that selectively prevent the release of azurophil granule contents without interfering with specific granule secretion, namely replacement of $\mathrm{Cl}^{-}$ with gluconate ${ }^{-}$or the addition of zinc. Replacement of $\mathrm{Cl}^{-}$was found to impair the pinocytosis process itself, whereas $\mathrm{ZnSO}_{4}$ appeared to prevent the fusion between endosomes and azurophil granules. These data support the concept that the (lysosomal type) azurophil granules, but not the specific granules, are secreted through the endosomal pathway. (J. Clin. Invest. 1994. 93:247-255.) Key words: lysosomes • ionophores • zinc • gluconates $\bullet$ signal transduction
\end{abstract}

\section{Introduction}

Neutrophils contain two main granule types called azurophil and specific granules by some (1) or primary and secondary granules by others (2). Although their secretion is thought to be regulated differentially, based on different release kinetics (3-5) and granule-selective secretagogues $(6,7)$ the intracellular signal(s) and transport mechanism(s) that control the selectivity of the secretion are poorly understood. Signals reported to play a role in neutrophil secretion "in general" such as the phosphorylation of heterotrimeric $G$ proteins ( 8$)$, the activity of protein kinase $C(9)$ or cytoplasmic Ca transients (10) have been linked unequivocally only to the release of specific granules. Thus, stimuli that activate these signals such as chemoattractants, phorbol esters, and $\mathrm{Ca}$ ionophores, elicit only specific

Address correspondence and reprint requests to Dr. Claus Fittschen, Pharmakologische Forschung, Abt. Spez. Pharmakologie, E. Merck, D-6100 Darmstadt, Germany.

Received for publication 31 December 1992 and in revised form 12 August 1993

J. Clin. Invest.

(C) The American Society for Clinical Investigation, Inc.

0021-9738/94/01/0247/09 \$2.00

Volume 93, January 1994, 247-255 granule release. Curiously, pretreatment of neutrophils with cytochalasin B (CB $)^{1}$ allows the same stimuli that alone elicit only specific granule release to induce secretion of both granule types $(11,12)$. Thus, CB provides an additional, so far unidentified, change in the cell physiology that is involved in azurophil granule secretion. Convincing evidence for distinct intracellular signaling pathways for the secretion of either granule type has been provided by Wilson et al. (9), who found that inhibition of protein kinase $\mathrm{C}$ with sphingoid bases impaired only the secretion of specific granules. Others have focussed on the role of $\mathrm{Ca}$ transients and suggested that specific granules are sensitive to low cytoplasmic $\mathrm{Ca}$ transients, whereas azurophil granules require high concentrations of intracellular $\mathrm{Ca}$ for secretion (13). Recently, several low molecular weight $\mathrm{G}$ proteins have been reported to bind to both granule types in a granule-specific manner (14). Despite these interesting findings of multiple intracellular targets of GTP-binding proteins (see also Barrowman et al. [15]), it is still unclear how these proteins might function in the differential secretion in neutrophils.

We have previously attempted to identify intracellular processes involved in azurophil granule secretion by studying the mechanism of action of the $\mathrm{Na}$ ionophore Monensin that elicits selectively the release of this granule type. Instead of binding to cell surface receptors activating the early cell signal cascade, Monensin appears to act directly on the membranes of azurophil granules, triggering their alkalinization, swelling, and secretion (7). In subsequent studies, and those reported herein, Monensin-treated neutrophils were found to form endosomes (pinosomes) that fused with azurophil granules, resulting in the formation of large vacuoles that contained the azurophil granule marker peroxidase. We reasoned that a connection of this lysosomal granule type to the endosomal system might represent a transport pathway for granule content to the outside, as also suggested earlier $(16,17)$. If that were true, azurophil-endosome fusion should be a prerequisite for the exocytosis of azurophil granule contents. To probe this possibility more effectively, we took advantage of our recent observations that replacement of all buffer chloride $(\mathrm{Cl})$ with gluconate ions or addition of zinc ions, each selectively inhibited azurophil granule secretion. If our hypothesis was correct, these treatments should impair not only azurophil granule secretion, but also one or several of the following processes: $(a)$ endosome internalization, $(b)$ endosome-azurophil granule fusion, or $(c)$ transport of endosomes or endosome-granule fusion products back to the plasma membrane. The results of this work are reported below.

1. Abbreviations used in this paper: $\mathrm{CB}$, cytochalasin $\mathrm{B} ; \mathrm{LDH}$, lactate dehydrogenase; LY, Lucifer yellow; MPO, myeloperoxidase; OpsZy, opsonized zymosan; Vit $\mathrm{B}_{12} \mathrm{BP}$, vitamin $\mathrm{B}_{12}$-binding protein. 


\section{Methods}

Chloride buffer ( $\mathrm{pH} 7.4$ ) was prepared to contain $135 \mathrm{mM} \mathrm{NaCl}, 4.8 \mathrm{M}$ $\mathrm{KCl}, 0.93 \mathrm{mM} \mathrm{CaCl}_{2}$, and $1.2 \mathrm{mM} \mathrm{MgCl}_{2}$ (all from Mallinckrodt, Paris, KY) as previously described for Krebs-Ringer buffer (18); the phosphate salts were replaced with $10 \mathrm{mM}$ Hepes (Sigma Chemical Co., St. Louis, MO). This buffer was supplemented to contain $0.2 \%$ dextrose (Abbott Laboratories, North Chicago, IL) and $0.25 \%$ low endotoxin HSA (Biocell Laboratories, Carson, CA). Gluconate buffer was prepared to contain concentrations of $\mathrm{Na}-, \mathrm{K}-, \mathrm{Ca}-$, and $\mathrm{Mg}$-gluconate that were equivalent to the chloride salts of the complete (chloride) buffer, dextrose (Sigma Chemical Co.) and HSA. The osmolality was adjusted to $290 \pm 5 \mathrm{mosmol} / \mathrm{kg}$. For zinc experiments $3 \mathrm{mM}$ $\mathrm{ZnSO}_{4}$ (Fisher Scientific, St. Louis, MO) was added to chloride buffer, which subsequently was readjusted to $\mathrm{pH} 7.35$. Neutrophils were suspended in this solution just before stimulation. Similar findings of inhibition as those described for $\mathrm{ZnSO}_{4}$ were obtained with $\mathrm{ZnCl}_{2}$ which, however, was not routinely used because the available commercial products were contaminated with toxic oxychlorides.

Stock solutions of $10^{-3} \mathrm{M}$ FMLP (Vega Biotechnologies, Tuczon, $\mathrm{AZ}$ ) and of $\mathrm{CB}$ at $5 \mathrm{mg} / \mathrm{ml}$ (Sigma Chemical Co.) were prepared in DMSO (Sigma Chemical Co.) and stored at $-18^{\circ} \mathrm{C}$. After dilution into buffer, FMLP was present at $10^{-7}$ and $C B$ at $2.5 \mu \mathrm{g} / \mathrm{ml}$. Monensin (Calbiochem Corp., San Diego, CA) was dissolved in ethanol (at 20 $\mathrm{mM}$ ) and stored at $4^{\circ} \mathrm{C}$; working solutions were made fresh before use (final concentration $=20 \mu \mathrm{M}$ ). Zymosan (Koch-Light Ltd., Havenhill, United Kingdom) was opsonized in fresh human serum, washed three times and used at a final particle/neutrophil ratio of $50: 1(3)$.

Human neutrophils were isolated by the plasma-Percoll method of Haslett et al. to be minimally activated or primed (18). These were consistently $>95 \%$ pure and viable.

\section{Assay procedures}

Neutrophil secretion was elicited in neutrophils $\left(10^{7}\right.$ cells $\left./ \mathrm{ml}\right)$ using $10^{-7} \mathrm{M}$ FMLP in the presence or absence of $2.5 \mu \mathrm{g}$ cytochalasin $\mathrm{B} / \mathrm{ml}$ buffer (preincubation $10 \mathrm{~min}$ ), or by using $2 \times 10^{-5} \mathrm{M}$ Monensin, or opsonized zymosan at a particle/cell ratio of 50:1 (the latter two stimuli without $\mathrm{CB}$ ). The supernate was assayed for myeloperoxidase (MPO) (19), vitamin $B_{12}$-binding protein (Vit $B_{12} B P$ ) (20) and lactate dehydrogenase (LDH) (19). Secretory activity was defined as the enzyme activity in the neutrophil supernate expressed in percent of cell content of neutrophil aliquots lysed with Triton X-100. LDH release, determined as indicator of cell injury (19), never exceeded $5 \%$ of the cell lysate.

\section{Flow cytometry}

Lucifer yellow (LY) fluorescence was estimated using cytofluorograph (Epics 751; Coulter Corp., Hialeah, FL) with a Biosense tip to increase its sensitivity and a Coherent argon ion laser (model 90-5; Innova, Palo Alto, CA). The system was connected to a computer system (Cicero; Cytomation, Inc., Englewood).

Cell suspensions exposed to stimuli or buffer in the presence of 0.5 $\mathrm{mg} \mathrm{LY} / \mathrm{ml}$ were cooled rapidly by dilution with 4 vol of ice cold buffer, followed by three washes with ice-cold buffer and fixation with paraformaldehyde (final concentration-1\%). Excitation at $488 \mathrm{~nm}$ resulted in green fluorescence that was measured using a 20-nm bandpass at 525 $\mathrm{nm}$. Responses to stimuli were recorded as changes in mean fluorescence of the cell population.

\section{Fluorescence microscopy}

Lucifer Y-labeled neutrophils fixed in 1\% paraformaldehyde (Electron Microscope Sciences, Fort Washington, PA), as described above, were applied in a $200-\mu 1$ drop to the center of a $35-\mathrm{mm}$ tissue culture dish (Falcon Labware, Oxnard, CA) and allowed to settle. After replacement of the buffer with a drop of high viscosity immersion oil photomicrographs were taken on black and white film (Pan-X-400; Kodak Corp., Rochester, NY) using a camera system ( Vario-Orthomat; Leitz, Wetzlar, Germany) attached to a Diaplan (Leitz) microscope.

\section{Uptake of ${ }^{125}$ I-BSA and cell fractionation}

Neutrophils were divided into two equal aliquots of $3-4 \times 10^{8}$ cells each and washed twice with chloride or gluconate-buffer as appropriate. These aliquots were again subdivided into three equal parts of $1-1.3 \times 10^{8}$ cells, to each of which $50 \mu \mathrm{Ci}$ of ${ }^{125} \mathrm{I}$-BSA (New England Nuclear, Boston, MA) was added. Paired samples were then incubated at $37^{\circ} \mathrm{C}$ in $3 \mathrm{ml}$ of gluconate or chloride buffer containing FMLP $\left(10^{-7}\right.$ $\mathrm{M}), \mathrm{CB} / \mathrm{FMLP}\left(2.5 \mathrm{mg} / \mathrm{ml}\right.$ per $\left.10^{-7} \mathrm{M}\right)$, or Monensin $\left(2 \times 10^{-5} \mathrm{M}\right)$ as described for the secretion assays. The neutrophils were cooled on ice, washed $3 \times$ in cold chloride or gluconate buffer, homogenized, and fractionated on a $34-60 \%$ sucrose gradient as described by West et al. (21). Markers for azurophil granules, specific granules and membrane fractions were MPO, Vit $\mathrm{B}_{12} \mathrm{BP}$, and alkaline phosphatase (21).

\section{Phagocytosis, isolation of phagolysosomes}

Phagocytosis of zymosan. Neutrophils and freshly opsonized zymosan particles were washed twice in chloride or gluconate buffer, as appropriate and resuspended in the following buffers (containing $0.25 \%$ HSA ): $(a)$ chloride buffer, $(b)$ gluconate buffer, and $(c)$ chloride buffer containing $3 \mathrm{mM} \mathrm{ZnSO}_{4}$. Cells and zymosan were combined at a particle: cell ratio of $5,10,33$, and $100: 1$ and incubated in duplicate at $37^{\circ} \mathrm{C}$ in a waterbath ( total vol $=500 \mu \mathrm{l} / \mathrm{vial}$ ). After $60 \mathrm{~min}, 20-\mu \mathrm{l}$ suspension were transferred from each vial into $500 \mu$ l glutaraldehyde $(0.2 \%$ in $0.9 \% \mathrm{NaCl})$ and examined for phagocytic activity. 50 cells/vial (100 cells/group) were evaluated and divided into those that had taken up 0-4 particles, 5-8 particles, or $\geq 9$ particles, respectively.

Isolation of phagolysosomes. $200 \times 10^{6}$ neutrophils each were transferred into two $50-\mathrm{ml}$ polypropylene tubes and washed twice with chloride buffer or gluconate buffer, as appropriate. These suspensions were again subdivided into three equal aliquots, centrifuged, and resuspended in chloride or gluconate buffer, respectively, containing $0.25 \%$ HSA. $60 \times 10^{6}$ neutrophils/vial in a volume of $5 \mathrm{ml}$ were mixed with 5 $\mathrm{ml}$ of a suspension of opsonized zyomosan particles ( final particle/cell ratio $=100: 1$ ), which had been washed $3 \times$ with chloride or gluconate buffer, respectively. After incubation for $60 \mathrm{~min}$ at $37^{\circ} \mathrm{C}$, the tubes were mixed well by inversion and $20 \mu \mathrm{l}$ suspension was transferred into $50 \mu \mathrm{l}$ glutaraldehyde to be evaluated for phagocytic activity, as described above. The remainder of the (unfixed) cell/zymosan suspension was cooled on ice for $15 \mathrm{~min}$ and centrifuged for another $10 \mathrm{~min}$ at $170 \mathrm{~g}$ and $4^{\circ} \mathrm{C}$. The supernate was completely removed, centrifuged again at $1,500 \mathrm{~g}$ and retained for analysis of MPO activity. The cell/zymosan mixture was resuspended in $4 \mathrm{ml}$ of ice-cold homogenization buffer containing $20 \mathrm{mM}$ Imidazole $/ \mathrm{HCl}, \mathrm{pH} 7.4,0.25 \mathrm{M}$ sucrose, 5 mM EGTA, and $2.5 \mathrm{mM} \mathrm{MgCl}$ and homogenized on ice in the cup of a sonifier cell disruptor (Branson Ultrasonics Corp., Danbury, CT) at setting 7 for $2 \mathrm{~min}$. Using this method, $96-98 \%$ of the neutrophils were found to be broken, as determined by counting cell numbers before and after sonication. Each of the six homogenates was loaded onto a cold, discontinuous Percoll gradient, in which the light layer consisted of $60 \%$ Percoll $/ 40 \%$ of $10 \times$ Imidazole buffer, and the heavy layer consisted of $90 \%$ Percoll $/ 10 \%$ of $10 \times$ Imidazole buffer (22). Centrifugation for $10 \mathrm{~min}$ at $1,500 \mathrm{~g}$ and $4^{\circ} \mathrm{C}$ resolved the homogenate into three fractions; i.e., the supernate containing the granule fractions, the $60 \%$ Percoll layer containing the nuclear fraction, and the $90 \%$ Percoll layer containing zymosan particles and phagolysosomes as described (22). The fractions were successively aspirated and their volumes recorded. After addition of Triton X-100 (final concentration $=0.2 \%$ ) the MPO activity was measured in each fraction (and in the supernate retained from the neutrophil-zymosan incubation). The total MPO content of the specimen was calculated by first multiplying the MPO activities in the three fractions and the cell supernate with their respective volumes, and then adding these together. The MPO activity of each fraction/supernate was then expressed as a percentage of this total MPO content.

\section{Electron microscopy/cytochemistry}

For ultrastructural studies, neutrophils were stimulated as described above, fixed in equal volumes of prewarmed glutaraldehyde ( $1.5 \%$ in 

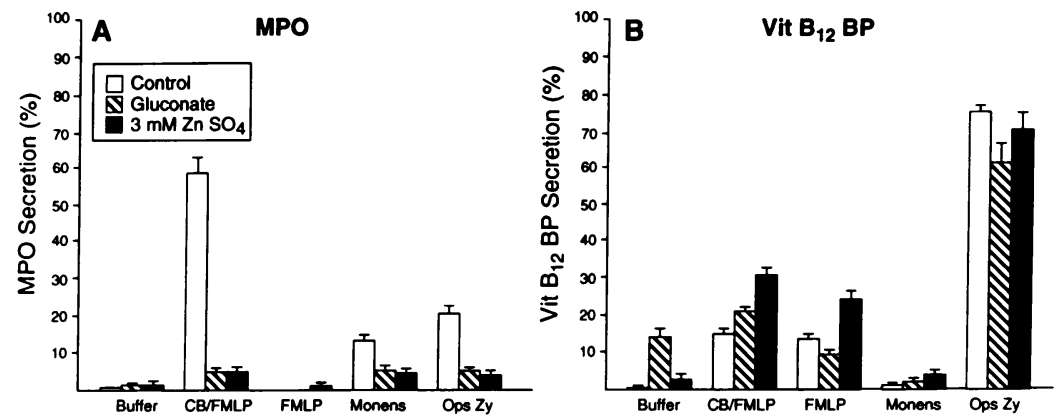

Figure 1. Neutrophil secretion of the azurophil granule marker MPO $(A)$ and of the specific granule marker Vit $\mathbf{B}_{12}$ BP $(B)$ in chloride buffer (open bars, controls) in chloride-free gluconate buffer, (hatched bars), and in the presence of $3 \mathrm{mM} \mathrm{ZnSO}_{4}$ (filled bars). Neutrophils were incubated as detailed in Methods in the presence of FMLP with and without pretreatment in CB, in presence of Monensin, and opsonized zymosan (Ops $Z y$ ), respectively. Bars indicate the mean \pm SEM of four experiments.

$0.1 \mathrm{M} \mathrm{Na}$-cacodylate buffer, $\mathrm{pH} 7.3$ ) at $37^{\circ} \mathrm{C}$ for $5 \mathrm{~min}$ and allowed to cool on ice for an additional $60 \mathrm{~min}$. Subsequently, the cells were washed three times in $0.1 \mathrm{M}$ cacodylate buffer and refrigerated overnight. Peroxidase activity was demonstrated using 3,3'-diaminobenzidine as substrate as described by Graham and Karnovsky (23). For easier transfer during dehydration procedures, neutrophils were pelleted and mixed with low gelling temperature agarose (Seaplaque; FMC Corp., Rockland, ME). The gel matrix containing the cells was then routinely dehydrated through graded acetones and propylene oxide and embedded in LX 112-Araldite 502 plastic (Ted Pella Inc., Redding, CA). Thin sections were viewed unstained (or when needed, stained with uranyl acetate-lead citrate) in a transmission electron microscope (model 400; Phillips Electronic Instruments Co., Mahwah, $\mathrm{NJ}$ ) at an accelerating voltage of $60 \mathrm{kV}$.

Endocytic activity, at the ultrastructural level, was visualized using cationized ferritin (at $1 \mathrm{mg} / \mathrm{ml}$ ) from Electron Microscope Sciences. Cell suspensions had a final volume of $1 \mathrm{ml}$ and a concentration of $10^{7}$ neutrophils $/ \mathrm{ml}$. Cells exposed to Monensin (and their buffer controls) were fixed after $45 \mathrm{~min}$, those stimulated with CB/FMLP after $11 \mathrm{~min}$ ( 10 min CB +1 min FMLP), CB controls after 11 min, FMLP controls after $10 \mathrm{~min}$ in buffer, and $1 \mathrm{~min}$ FMLP, accordingly. The preparations were fixed in suspension with a 10 -fold excess of prewarmed $\left(37^{\circ} \mathrm{C}\right)$ glutaraldehyde ( $1.5 \%$ in $0.1 \mathrm{M} \mathrm{Na}$-cacodylate buffer) and processed as described above.

\section{Results}

Secretion studies. As depicted in Fig. 1, in buffer containing chloride ions, neutrophils stimulated with CB/FMLP and zymosan secreted both the azurophil granule contents (here indicated by MPO) and specific granules (Vit $\mathrm{B}_{12} \mathrm{BP}$ ). Cells exposed to the $\mathrm{Na}$ ionophore Monensin secreted only MPO, a process we previously suggested as a model for the exocytosis of azurophil granules (7). By contrast, FMLP in the absence of $\mathrm{CB}$ induced only specific granule secretion. These stimulus combinations, therefore, were used to probe the differential mechanisms of azurophil and specific granule exocytosis.

In the absence of chloride ions (i.e., gluconate buffer), neutrophil MPO secretion in response to all three stimuli was markedly impaired, whereas $\mathrm{Vit} \mathrm{B}_{12} \mathrm{BP}$ release remained intact (Fig. 1). (In fact, even in the absence of stimulus, gluconate buffer controls consistently demonstrated some release of Vit $\mathrm{B}_{12} \mathrm{BP}$ that never was associated with $\mathrm{LDH}$ release and, thus, was not caused by a toxic effect. This observation, which could be of interest for studies of specific granule secretion, is currently unexplained.) While no preincubation was necessary for the prevention of azurophil granule secretion by chloride deprivation, the cells had to be washed twice in gluconate buffer to completely remove the extracellular $\mathrm{Cl}$. The concentration dependence of this secretion on chloride ions is shown in Fig. 2, wherein it was found that small amounts of chloride in the buffer had disproportionate permissive effects on MPO secretion. Thus the inability of previous studies (24) to demonstrate the importance of $\mathrm{Cl}^{-}$for the secretion of azurophil granules may have been related to the presence of residual $\mathrm{Cl}$ ions. In contrast to chloride, sulphate ions $\leq 30 \mathrm{mM}$ were unable to support azurophil granule secretion (Fig. 2). This sulphate concentration was several-fold that usually present in biological salt solutions indicating that extracellular sulphate is not important for the secretory process under investigation.

An inhibitory pattern similar to that caused by $\mathrm{Cl}$ replacement with gluconate has recently been documented for the addition of zinc ions ( 7 ) and these data are also included in Fig. 1 for comparison. The observations support the supposition that the secretory mechanism(s) involved in azurophil granule secretion can be separated from that (those) facilitating specific granule secretion. Accordingly, using these two inhibitory principles, the following studies examined the possibility that the endocytic pathway was involved in secretion of azurophil granule contents.

Association of azurophil granule secretion with endocytosis? One of the most striking ultrastructural changes in neutrophils treated with Monensin as well as with CB/FMLP consists of the presence of large, round or elongated cytoplasmic vacuoles of which many contain peroxidase-positive material ( references 16, 25, and Fittschen, C., unpublished observation ). Hypothesizing that these organelles may represent endosomes resulting from increased endocytic activity induced by such stimuli, neutrophils were exposed to CB/FMLP or Monensin in the presence of the endosomal marker LY to estimate the cells' endocytic activity. Both secretagogues induced marked

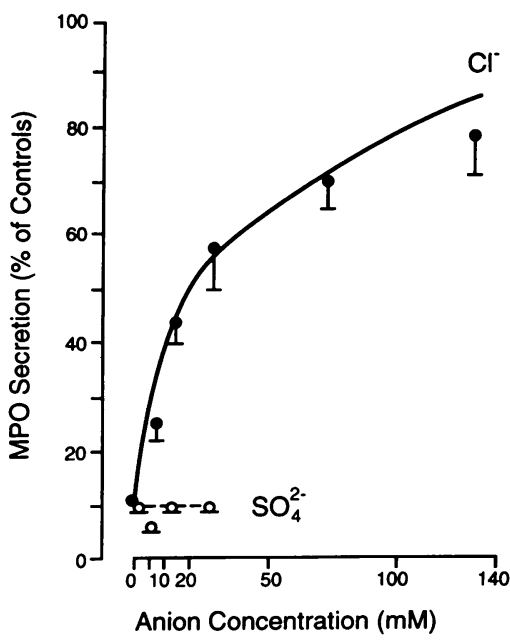

Figure 2. Chloride dependence of MPO secretion. Neutrophils washed twice with gluconate buffer were suspended in gluconate buffer containing the indicated concentrations of $\mathrm{Cl}^{-}$and $\mathrm{SO}_{4}^{2-}$ Only $\mathrm{Cl}^{-}$supported MPO secretion elicited by FMLP after pretreatment with CB. Each point indicates the mean \pm SEM of three experiments. 
fluid phase uptake into vacuoles as indicated by a punctate staining pattern on fluorescence microscopy that was not apparent in the unstimulated cells. Using cytofluorography, it was determined that in both time-course and volume endocytosed (Fig. 3) the uptake was proportional to the secretory activity already shown to be elicited by these agents (7). Importantly, the replacement of buffer $\mathrm{Cl}$ with gluconate severely impaired this fluid phase uptake, again in a quantitative and temporal pattern similar to the inhibition of azurophil granule secretion. This observation suggested that the whole endocytic process was impaired in the absence of chloride.

By contrast, addition of $\mathrm{ZnSO}_{4}$ did not inhibit pinocytosis of LY induced by Monensin or CB/FMLP (Fig. 4). $\mathrm{ZnSO}_{4}$ by itself induced some pinocytic activity that appeared additive with the uptake stimulated by Monensin.

Taken together, these findings indicate: $(a)$ that the same stimuli that caused azurophil granule secretion also induced fluid endocytosis; $(b)$ that replacement of buffer $\mathrm{Cl}$ with gluconate inhibited secretion of azurophil (but not of specific) granules as well as fluid phase endocytosis, consistent with a causal relationship between both processes; $(c)$ that while zinc did inhibit azurophil granule secretion, it presumably acted in some way different from the chloride deprivation.

Secretion of preloaded endosomal markers. Interconnection of endosomes with azurophil granules as part of the exocytic process would imply that a secretory stimulus should elicit not only uptake of endosomal markers, but also, along with the secretory process, their release back to the extracellular medium. Accordingly, the neutrophil endosomal system (and presumably a proportion of azurophil granules) was preloaded with LY by exposure to Monensin before induction of a secondary release reaction with CB/FMLP (Fig. 5 ). Not surprisingly, some dye was released without further stimulation presumably reflecting the descending limb of endosomal transcytosis. However, the release was doubled by the addition of CB/FMLP, further supporting the suggestion of a link between pinocytosis

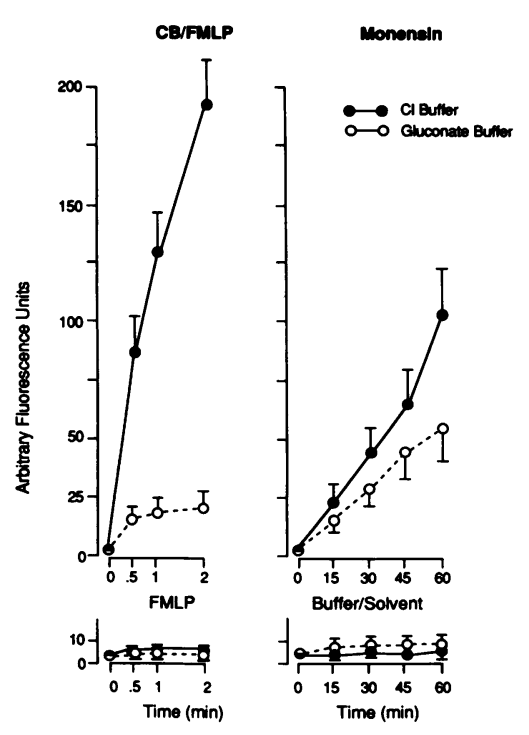

Figure 3. Endocytosis of LY by neutrophils stimulated in the presence of $\mathrm{Cl}$ buffer (closed symbols) and $\mathrm{Cl}^{-}$-free gluconate buffer (open symbols). Cells were stimulated with FMLP (lower left panel), FMLP after pretreatment with CB (upper left panel), monensin (upper right), and solvent $(0.1 \%$ ethanol $)$ controls for the monensin experiments (lower right panel). Buffer controls for FMLP and CB controls for $\mathrm{CB} /$ FMLP demonstrated less activity than measured in the presence of FMLP alone (not shown). Each point indicates the mean \pm SEM of three experiments.
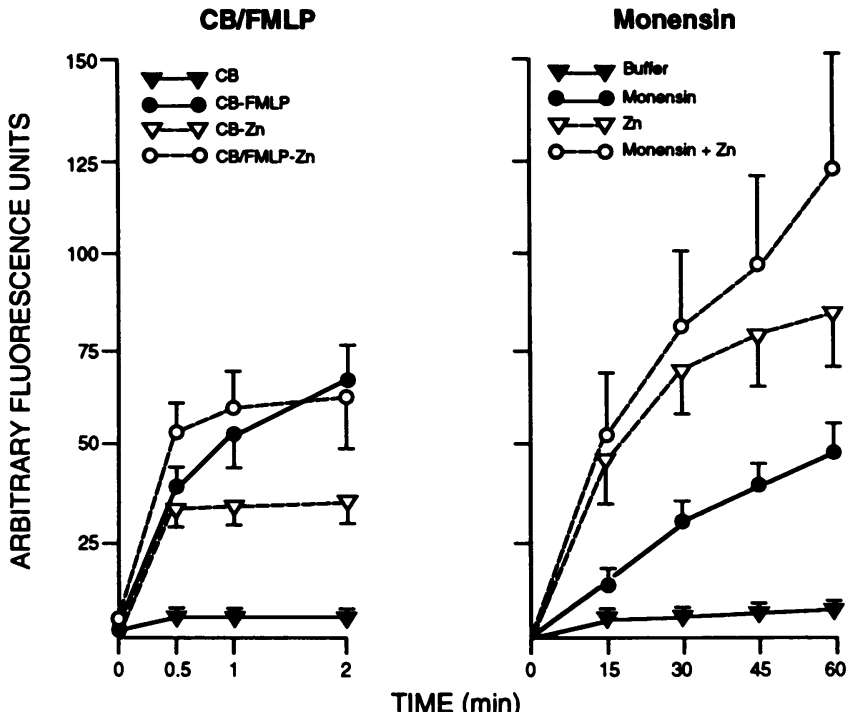

Figure 4. Endocytosis of LY in the absence (filled symbols) and presence (open symbols) of $3 \mathrm{mM} \mathrm{ZnSO}_{4}$. Neutrophils were stimulated as indicated in Fig. 3. Controls exposed to buffer or FMLP alone (with and without $\mathrm{ZnSO}_{4}$ ) were indistinguishable from $\mathrm{CB}$ and $\mathrm{CB}$ $\mathrm{Zn}$, respectively. Data represent the mean $\pm \mathrm{SEM}$ of three experiments.

and azurophil granule secretion. A similar effect was seen if the order of the stimuli was reversed (data not shown).

Fusion of endosomes with azurophil granules. The question of endosome/granule fusion was examined both by electron microscopy and by subcellular fractionation. For ultrastructural studies, neutrophils were stimulated after addition to the buffer of an electron dense marker of endosomes, cationized ferritin. In addition to its presence in the fluid medium this positively charged label is enriched at the negatively charged neutrophil plasma membrane, providing a sensitive marker for any endocytic activity. Neutrophils treated with Monensin or CB/FMLP contained ferritin in variably sized electron lucent vacuoles. These vacuoles usually were 2-10 times larger than azurophil granules, and often included the azurophil granule marker peroxidase (Fig. 6). This confirmed derivation of the vacuoles from endosomes and that these endosomes also fused

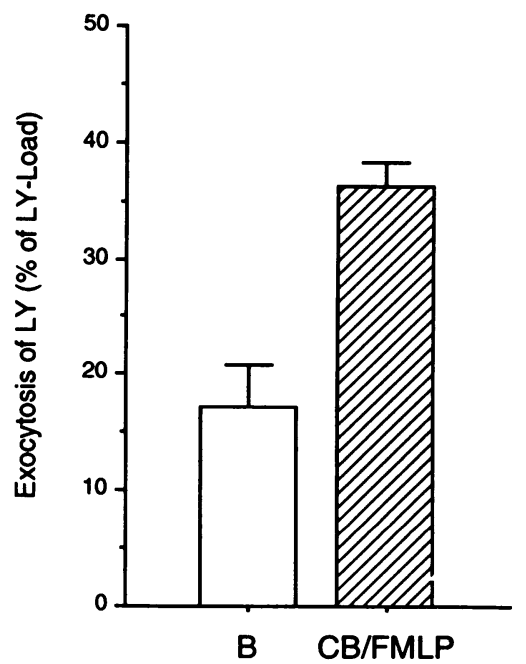

Figure 5. Neutrophil exocytosis of endocytosed LY in response to CB/FMLP. Cells were preloaded with $L Y$ by incubation for 45 $\min \left(37^{\circ} \mathrm{C}\right)$ with $10^{-5}$ Monensin (M). After two ice-cold washes with buffer the neutrophils were reincubated at $37^{\circ} \mathrm{C}$ in buffer which resulted in a basal release $(B)$, that was enhanced when the cells were treated for $5 \mathrm{~min}$ with CB $(2.5 \mu \mathrm{g} / \mathrm{ml})$ followed by $1 \mathrm{~min}$ with $10^{-7}$ FMLP ( $C B$ ) $F M L P)$. Results shown are the means of three experiments \pm SEM. 

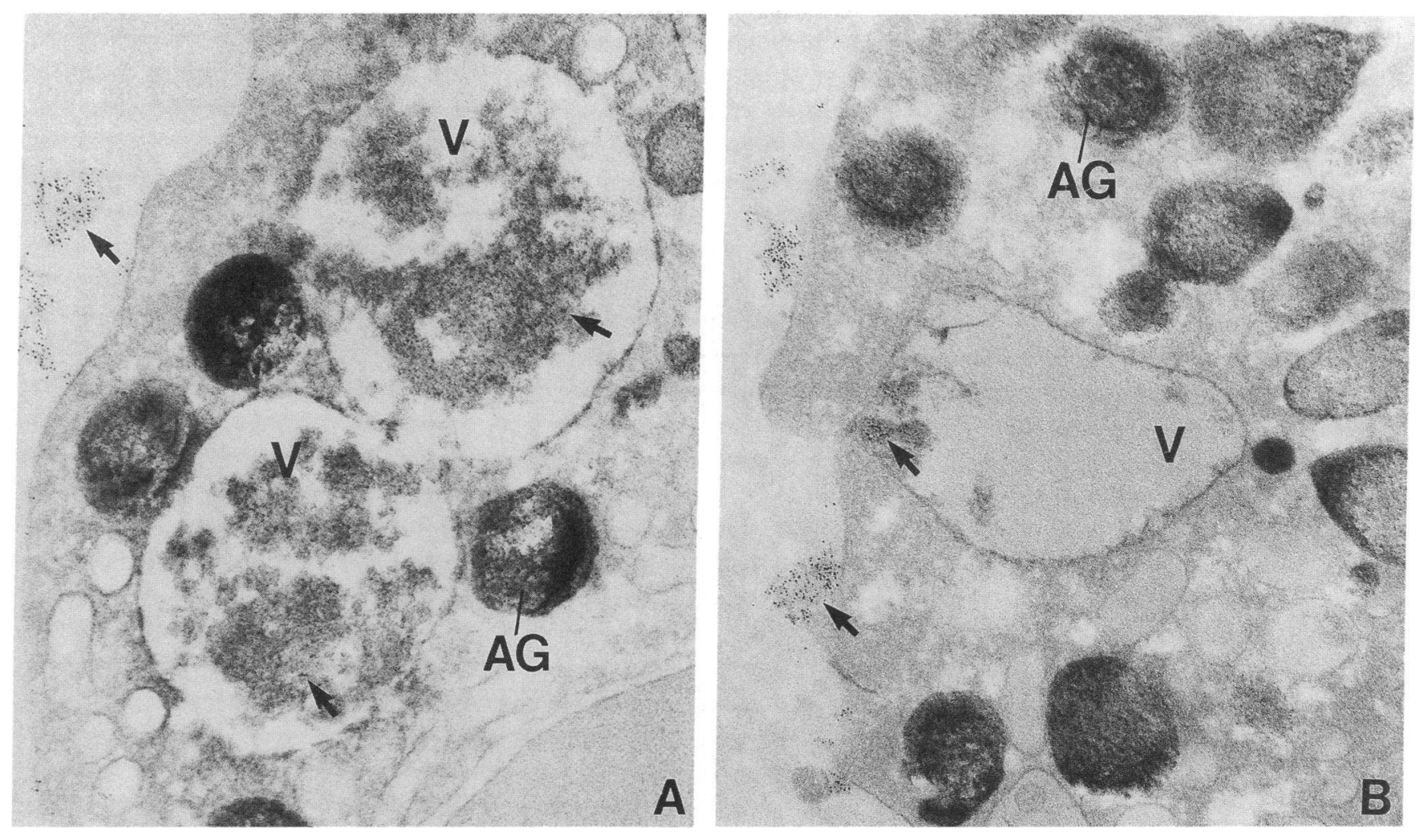

Figure 6. Colocalization of endocytosed cationized ferritin and MPO in vacuoles derived from the fusion of endosomes and azurophil granules. Neutrophils were incubated in ferritin-containing buffer with and without stimuli (see Methods). Only neutrophils treated with Monensin $(A)$ or CB/FMLP $(B)$ (but not buffer controls or cells treated with CB or FMLP, respectively) showed ferritin and MPO in the same organelles. V, vacuoles containing MPO (fine black reaction product) and coarse ferritin grains (arrows); AG, azurophil granules. $\times 52,000$. Peroxidase without counterstain; glutaraldehyde with $\mathrm{O}_{5} \mathrm{O}_{4}$ postfixation.

with azurophil granules. Control cells in the absence of secretagogues demonstrated no association of ferritin with azurophil granules vacuoles, although some smaller ferritin-containing endosomes (no larger than azurophil granules) were sometimes present within $1.5 \mu \mathrm{m}$ beneath the plasma membrane.

To examine endosome-azurophil granule fusion more quantitatively, neutrophils were stimulated with FMLP, CB/ FMLP, or Monensin in the presence of the fluid phase marker ${ }^{125}$ I-BSA, followed by cell homogenization and density centrifugation. The subcellular distribution of ${ }^{125} \mathrm{I}$ in neutrophils is shown in Fig. 7 and Table I. Cells exposed to FMLP alone showed no measurable uptake of ${ }^{125}$ I-BSA into the granule fractions; the supernates (fractions 20-24), however, did contain some radiolabel. By contrast, neutrophils stimulated with either Monensin or CB/FMLP in chloride buffer had abundant label in the granule fractions. The marker was found in fractions containing the azurophil granules, as well as those of lower density. This distribution would be expected if the ${ }^{125} \mathrm{I}-$ BSA was in organelles representing fusion products of the dense azurophil granules and of endosomes, which are of much lower density. Label was also present in membrane and supernate fractions. Most of this radioactivity in the supernate was originally cell associated and not caused by insufficient washes, since it exceeded the ${ }^{125}$ I content present in equivalent volumes of the last cell wash by 5-10-fold. It may have originated from endosomes and/or endosome-granule fusion products that broke during the homogenization procedure, as suggested by the presence of MPO in the same fractions.
In the absence of chloride ions (gluconate buffer), neutrophils stimulated with either CB/FMLP or Monensin showed much less radiolabel in the granule fractions (Fig. 7 and Table I). This was in keeping with the inhibition of LY endocytosis observed in this buffer.

Importantly, zinc also reduced incorporation of ${ }^{125} \mathrm{I}-\mathrm{BSA}$ into the granule fractions after stimulation with CB/FMLP or Monensin (Fig. 7 and Table I). The inhibition was basically similar to that achieved with $\mathrm{Cl}$ replacement, although cell preparations treated with zinc frequently contained more label in the supernate fractions, consistent with enhanced endocytosis. Taken together with the experiments on LY endocytosis, these observations suggest that zinc, while not impairing endocytosis, does in fact inhibit fusion between endosomes and azurophil granules and raise the possibility that this is the mechanism for its inhibition of secretion.

In contrast to the link between fluid endocytosis and the secretion process studied here, the closely related association of particle uptake and secretion has been thoroughly studied previously. We, therefore, asked whether the conditions that modified granule-endosome interaction also interfered with phagocytosis and/or phagosome-azurophil granule fusion. In gluconate buffer, at low particle/cell ratio $(\leq 33: 1)$, neutrophil phagocytic activity was significantly lower ( $44 \%$ decrease \pm 3.5 , mean \pm SEM in three experiments) than in chloride buffer. At higher ratios (100:1), this effect was completely overcome, and phagocytic uptake was comparable in both conditions (see footnote, Table II). To examine whether $\mathrm{Cl}^{-}$-free conditions 

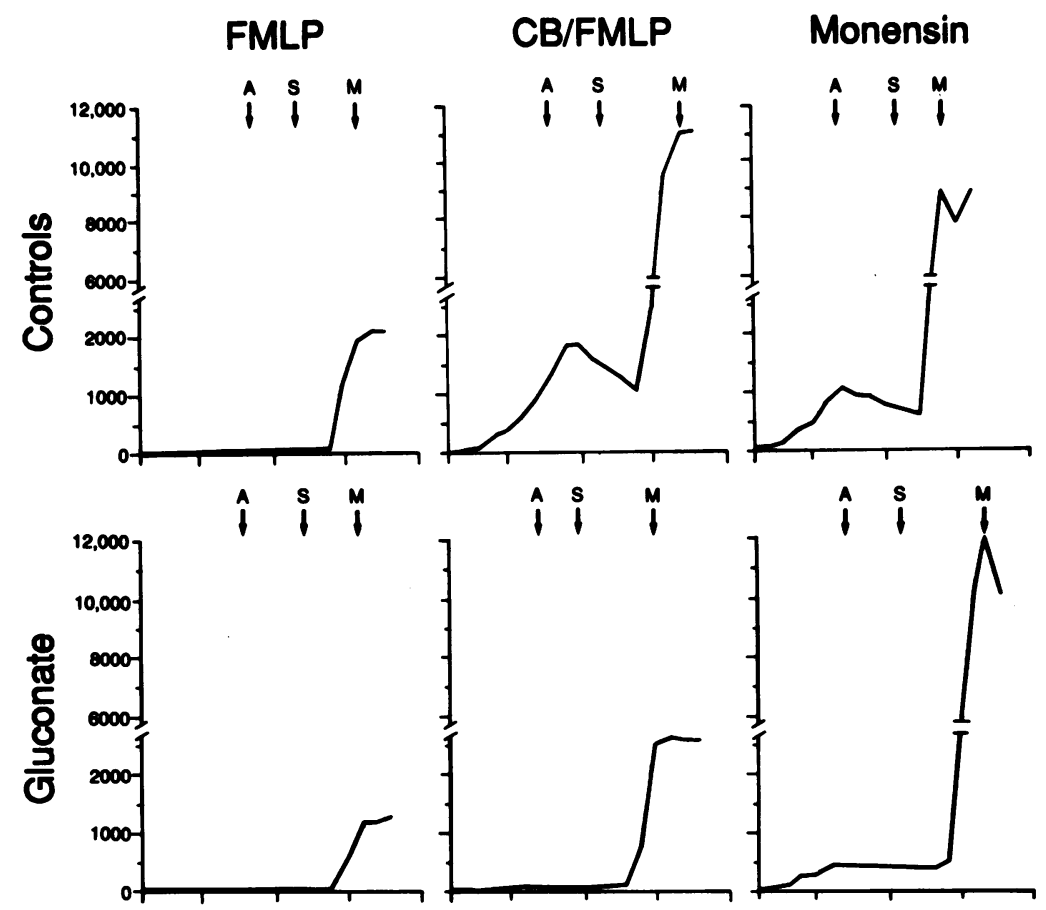

Figure 7. Fractionation of neutrophil homogenates after endocytic stimulation in $\mathrm{Cl}$ buffer, gluconate buffer and $\mathrm{Cl}$ buffer containing $3 \mathrm{mM} \mathrm{ZnSO}_{4}$. Stimulation with FMLP, CB/FMLP, and Monensin, respectively, in the presence of the endocytic marker

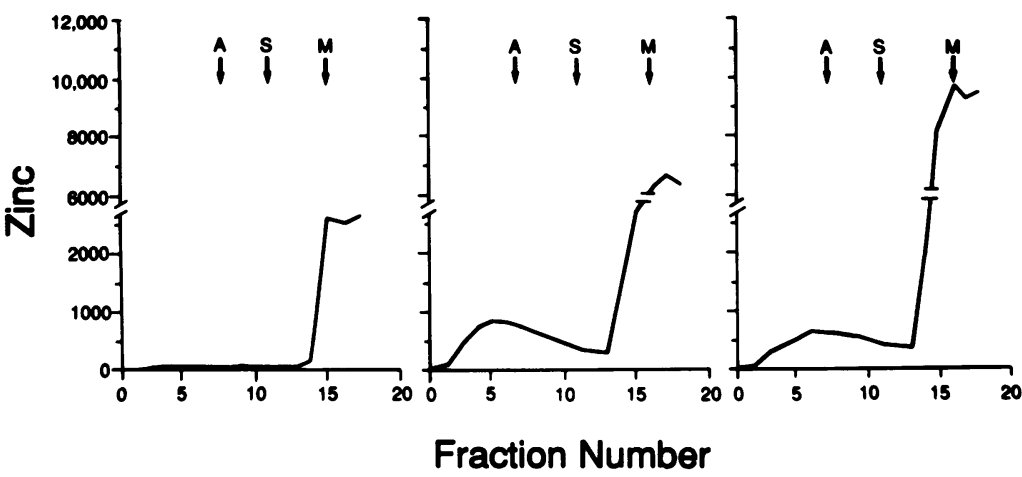

${ }^{125}$ I-BSA resulted in uptake of label. After homogenization and separation of the subcellular organelles by density centrifugation, label was found in membrane fractions (peak at $M$ ) and in granule fractions (A, peak of the azurophil granule marker MPO; , peak of the specific granule marker Vit $B_{12}$ BP). Neutrophils incubated in buffer or CB alone demonstrated similarly low activity as shown for FMLP. Cells stimulated in gluconate buffer or in the presence of $3 \mathrm{mM}$ $\mathrm{ZnSO}_{4}$ incorporated less ${ }^{125} \mathrm{I}$ into the granule fractions than did those stimulated in $\mathrm{Cl}$ buffer. This experimental result was representative of three similar experiments.

inhibited the fusion of phagosomes and azurophil granules, we isolated neutrophil phagosomes by cell fractionation using discontinuous Percoll gradients. Table II shows a comparison of the MPO activity in the phagosomal fraction obtained from neutrophils in gluconate buffer and in chloride buffer (particle $/$ cell ratio $=100: 1)$. It is apparent that the phagosomal fraction ( $90 \%$ Percoll) of cells incubated in gluconate buffer contained significantly less MPO than did the fraction of cells incubated in chloride buffer. The MPO activity in the nuclear fraction (60\% Percoll) may stem from contaminating phagosomes and/or MPO adsorbed to the nuclear material. This question was not further investigated. Thus, our results suggest that chloride ions may also be required for the fusion of phagosomes with azurophil granules.

Similar experiments with zinc were precluded by its inhibitory effect on phagocytosis ( $32 \%$ inhibition \pm 4 . 1 , mean \pm SEM in three experiments) even at a particle/cell ratio of 100:1.

\section{Discussion}

To be able to independently control secretion of two different granule populations, neutrophils must have either differentiating mechanisms in their intracellular signaling pathways or in their granule delivery to the plasma membrane or both. Recent evidence (9) suggests that the intracellular signals eliciting specific granule but not azurophil granule secretion include the action of protein kinase $\mathrm{C}$. Here, we provide supporting evidence that there may also be differences in the manner by which the contents of the granule is delivered to the outside and suggest that azurophil granule, but not specific granule, secretion occurs via endosomal organelles. Conceptually, this is an attractive possibility with some experimental support in other cell systems (17). The well-documented endosomal shuttle that moves material and membrane from the plasma membrane to lysosomes and back to the periphery provides an ideal means of transport for granule contents, particularly since the azurophil granule is the lysosome equivalent in this cell. Evidence in support of the suggested mechanism includes: $(a)$ Monensin, an azurophil granule-selective secretagogue (7) that does not act through receptor-associated mechanisms, induced fluid endocytosis (pinocytosis) concurrent with the secretion. (b) The Monensin-induced secretion was prevented by inhibition of either endocytosis per se, or of the observed fusion of endosomes with azurophil granules. $(c)$ In the case of a complete secretagogue (the combination of cytochalasin B and 
Table I. Fractionation of Neutrophils Stimulated to Pinocytose-soluble ${ }^{125}$ I-BSA; Uptake of Label into Granule Fractions*

\begin{tabular}{|c|c|c|c|c|c|}
\hline \multirow[b]{2}{*}{ Marker } & \multirow{2}{*}{\multicolumn{2}{|c|}{ Groups }} & \multicolumn{3}{|c|}{ Stimulants $^{\S}$} \\
\hline & & & FMLP & CB/FMLP & Monensin \\
\hline \multirow[t]{6}{*}{${ }^{125} \mathrm{I}(\mathrm{cpm})$} & Controls & $G^{\ddagger}$ & $751 \pm 182$ & $17,865 \pm 6,572$ & $7,713 \pm 697$ \\
\hline & & $\mathbf{M}$ & $3,356 \pm 853$ & $15,302 \pm 5,176$ & $19,789 \pm 5,794$ \\
\hline & Gluconate & G & $944 \pm 301$ & $2,386 \pm 480$ & $3,688 \pm 1,013$ \\
\hline & & $\mathbf{M}$ & $2,896 \pm 1,220$ & $7,462 \pm 2,767$ & $16,702 \pm 5,894$ \\
\hline & $\mathrm{ZnSO}_{4}$ & G & $764 \pm 339$ & $7,382 \pm 3,362$ & $3,723 \pm 2,479$ \\
\hline & & $\mathbf{M}$ & $6,367 \pm 3,339$ & $13,455 \pm 4,101$ & $17,703 \pm 8,335$ \\
\hline \multirow[t]{6}{*}{ MPO (arbitrary units) } & Controls & G & $58,396 \pm 6,803$ & $33,117 \pm 6,424$ & $44,679 \pm 6,001$ \\
\hline & & $\mathbf{M}$ & $10,745 \pm 1,940$ & $20,379 \pm 4,118$ & $19,608 \pm 6,588$ \\
\hline & Gluconate & G & $51,650 \pm 5,990$ & $51,472 \pm 8,828$ & $49,337 \pm 8,857$ \\
\hline & & $\mathbf{M}$ & $12,720 \pm 2,097$ & $17,506 \pm 3,897$ & $14,968 \pm 7,853$ \\
\hline & $\mathrm{ZnSO}_{4}$ & G & $52,186 \pm 14,080$ & $43,633 \pm 9,838$ & $45,978 \pm 11,283$ \\
\hline & & $\mathbf{M}$ & $7,418 \pm 1,474$ & $5,534 \pm 3,260$ & $14,825 \pm 3,115$ \\
\hline \multirow[t]{6}{*}{ Vit $B_{12} B P$ (arbitrary units) } & Controls & G & $41,196 \pm 6,105$ & $23,469 \pm 4,835$ & $31,544 \pm 6,977$ \\
\hline & & $\mathbf{M}$ & $13,747 \pm 5,977$ & $16,454 \pm 3,747$ & $15,742 \pm 3,235$ \\
\hline & Gluconate & G & $39,973 \pm 8,074$ & $39,514 \pm 6,354$ & $37,360 \pm 4,371$ \\
\hline & & $\mathbf{M}$ & $14,872 \pm 2,897$ & $10,537 \pm 2,216$ & $13,871 \pm 3,832$ \\
\hline & $\mathrm{ZnSO}_{4}$ & G & $41,986 \pm 8,817$ & $43,241 \pm 8,569$ & $39,056 \pm 5,385$ \\
\hline & & $\mathbf{M}$ & $9,135 \pm 2,920$ & $8,317 \pm 2,558$ & $11,748 \pm 6,141$ \\
\hline
\end{tabular}

* Results shown represent the mean \pm SEM of three experiments. ${ }^{\ddagger}$ Membrane fractions $(M)$ were identified using the marker enzyme alkaline phosphatase (not shown). The granule fractions (G) were defined as those lacking alkaline phosphatase activity. ${ }^{\S}$ Stimulants were used at concentrations indicated in Methods.

FMLP), inhibition of endocytosis or endosome-granule fusion prevented secretion of only azurophil granule contents; i.e., did not affect the release of specific granules.

Ligand-receptor interaction triggers endocytic activity in a variety of cells, including neutrophils. Endosomes thus formed transport ligand-receptor complexes and/or fluid to intracellular sites, such as lysosomes, where such complexes may be dissociated or degraded (26). However, neutrophils stimulated in

Table II. Fractionation of Neutrophil Homogenates after Zymosan Phagocytosis*‡

\begin{tabular}{lcc}
\hline \multicolumn{1}{c}{ Fraction } & Gluconate buffer & Chloride buffer \\
\hline Granule fraction (supernate) & $68.8 \pm 4.2$ & $38.5 \pm 6.1$ \\
Nuclear fraction (60\% Percoll) & $15.8 \pm 2.1$ & $18.5 \pm 3.8$ \\
Phagosomal fraction (90\% Percoll) & $3.2 \pm 1.0$ & $7.8 \pm 0.9$ \\
Neutrophil supernate (secretion) & $12.2 \pm 2.3$ & $35.2 \pm 3.4$ \\
Total & $100 \%$ & $100 \%$ \\
\hline
\end{tabular}

* Results show the distribution of MPO activity in percent of the total activity of the sample, and are expressed as the means \pm SEM of three experiments. ${ }^{\ddagger}$ Phagocytic activity ( 50 cells evaluated in duplicate per buffer condition and experiment).

Gluconate buffer: $0-4$ particles $=11.2 \%$ of neutrophil population (range $=6-18 \%$ )

$5-8$ particles $=84.4 \%($ range $=77-90 \%)$

$9+$ particles $=4.4 \%($ range $=0-8 \%)$

Chloride buffer: $\quad 0-4$ particles $=8.4 \%($ range $=4-15 \%)$

$5-8$ particles $=81.6 \%($ range $=77-84 \%)$

$9+$ particles $=10 \%($ range $=8-12 \%)$ the absence of CB with chemotactic ligands such as FMLP or C5a, complement fragment 5a, are known to internalize ligand-receptor complexes by a pathway that bypasses the azurophil granules (27). This process appears to be associated with uptake of only small amounts of fluid phase marker as seen with LY and ${ }^{125}$ I-BSA in this study. By contrast, stimulation of cells pretreated with $C B$ enhanced uptake of both LY and of ${ }^{125} \mathrm{I}$-BSA, i.e. markers of fluid phase endocytosis as also observed previously by others (28). This was accompanied by a marked amplification of azurophil granule exocytosis but had little effect in these studies on the release of specific granules. While the data are consistent with the hypothesis under examination, they appear at odds with previous reports that $\mathrm{CB}$ inhibits endocytosis in neutrophils (29). A significant difference between the systems, however, is that the studies reported herein used fluid phase markers, whereas the inhibitory effects of CB were on ligand uptake (29) (or on particles with ligands on them) (30). Therefore, it seems possible that FMLP can induce fluid phase pincytosis after pretreatment with $\mathrm{CB}$, even when the uptake of the FMLP-receptor complexes is reduced (31). The exact mechanism(s) by which cytochalasins enhance neutrophil responses is unknown, and there may indeed be more than one effect. The data reported here are consistent with a contribution to azurophil granule secretion through increased/altered endosomal activity.

The endosomal pathways within a given cell type appear to be quite complicated (26), and their driving forces and targeting mechanisms are still poorly understood. This complexity also implies that a number of sites within these pathways may be sensitive to modification and/or inhibition. In the model of endosome-linked secretion suggested here, these points could include $(a)$ endosome formation itself, $(b)$ transport of endosomes to azurophil granules, $(c)$ fusion between endosomes 
and granules, $(d)$ transport of endosome/granule fusion product back to the plasma membrane, and $(e)$ exocytosis of these at the plasma membrane (see model Fig. 8). In the present work, two separate steps within the transcellular pathway of neutrophil endosomes were found to be sensitive to modification. Thus, the formation of endosomes, identified by uptake of endosomal markers, appeared to be sensitive to $\mathrm{Cl}$ replacement with gluconate, an anion that is unable to substitute for $\mathrm{Cl}$ in most anion translocations across membranes $(32,33)$. It is unclear whether the $\mathrm{Cl}$ ions were required intracellularly or extracellularly, because in the absence of extracellular $\mathrm{Cl}$, cytoplasmic $\mathrm{Cl}$ is likely to leak out of the cell (down its concentration gradient) through an anion exchanger (33), presumably leading to a secondary intracellular $\mathrm{Cl}$ deficiency.

The next steps in the azurophil granule-endosome interaction are vesicle transport to the granules and the fusion of both organelles. One or both of these actions appear to be impaired in the presence of zinc. Endosomes, during their course through the cell, are acidified by an electrogenic proton ATPase (proton pump) $(32,34)$, and zinc is known to inhibit this activity (35). It is, therefore, an attractive possibility that is consistent with our findings that the proton pump may influence endosomal transport to and/or endosomal fusion with azurophil granules. Since the proton pump requires cotransport of $\mathrm{Cl}^{-}$for full activity (32), it seems likely that impairment of anion conductance (as is the case in gluconate buffer) contributes to the regulation of this step as well.

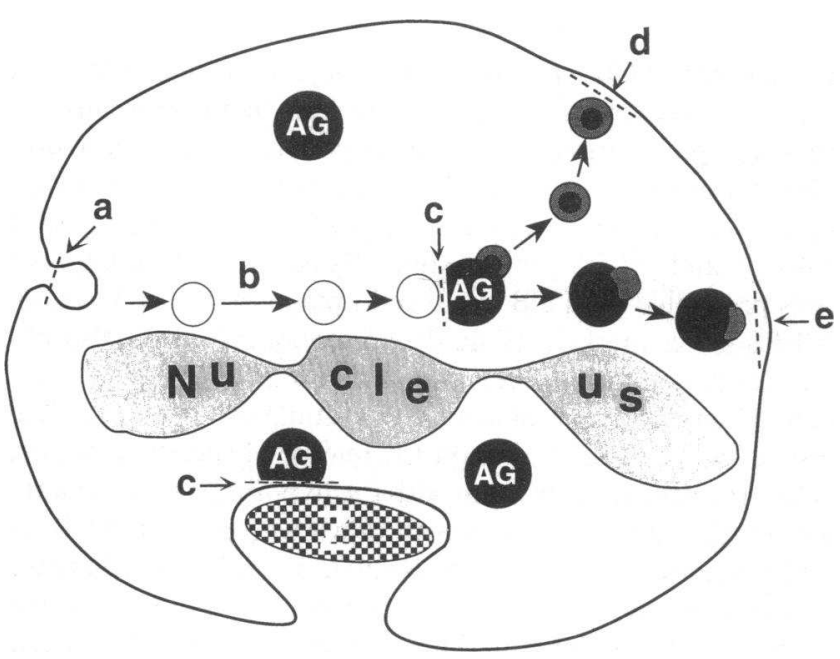

Figure 8 . This model summarizes the postulated interaction between azurophil granules and the endosomal cycle: upon appropriate stimulation endosomes are formed $(a)$, are transported to $(b)$, and fuse $(c)$ with azurophil granules $(A G)$, resulting in large azurophil vacuoles with flocculent content as frequently observed in neutrophils stimulated in vivo (see also reference 41 ) and in vitro. The endosomes may detach again and transport some granule content back to the plasma membrane $(d)$ or the whole vacuole may be translocated to the periphery $(e)$, perhaps using the endosomal membrane as contact site for recognition and fusion with the plasma membrane. In gluconate buffer, step $a$ is inhibited (and because of this, possible effects on step $c$ were not apparent), whereas $\mathrm{ZnSO}_{4}$ appears to impair steps $b$ and/or $c$. Inhibitors of step $d$ or $e$ are not yet known. Phagosomes undergo a similar interaction with azurophil granules (below nucleus): the phagosome forms enclosing the particle $\mathrm{Z}$, contacts and fuses with the granule. This fusion process, which conceptually corresponds to that in the endosomal pathway, was therefore also designated $(c)$ and is impaired in gluconate buffer.
The findings described above are consistent with recent studies in several cell types obtained from cystic fibrosis patients showing that endosomal transcytosis and acidification are influenced by $\mathrm{Cl}$ conductance through anion channels ( 36 , 37 ). It is, therefore, not unexpected that azurophil granule secretion was found to be impaired in neutrophils of cystic fibrosis patients (reference 38 and Fittschen, C., unpublished observation).

The observations that zinc and chloride replacement impaired not only azurophil granule secretion elicited by $\mathrm{CB} /$ FMLP and Monensin, but also that associated with zymosan phagocytosis prompted us to study also phagosome-granule interactions. Phagosomes have the advantage, $(a)$ that their fusion with azurophil granules is well established $(5,39)$ and $(b)$ that they are more easily isolated than endosomes, allowing us to address the question whether a treatment affected vesiclegranule fusion. Analogies in both processes were clearly apparent in chloride replacement studies: zymosan uptake was significantly decreased in gluconate buffer at low particle/cell ratios, presumably corresponding to the impaired fluid uptake observed with CB/FMLP and Monensin. When this inhibition was overridden at a high particle/cell ratio a second effect was identified, namely, that chloride replacement with gluconate downregulated phagosome-azurophil granule fusion. This requirement for chloride, one might speculate, could also be present in endosome-azurophil granule fusion. It may not be demonstrable in the fluid phase system, because the earlier step of endosome formation is so effectively blocked in the absence of chloride. Similar experiments could not be performed with zinc because of its consistent inhibitory effect on phagocytosis.

Although these observations suggest that the exocytosis of azurophil, but not of specific granule content, depends on the transcytosis of fluid phase endosomes, it is also clear that stimulation of fluid endocytosis by itself does not result in azurophil granule secretion. Thus, the endocytic response to CB/FMLP extended over a several-fold longer timespan than did the secretory phase. Moreover, PMA, a strong stimulus for fluid endocytosis elicits neither endosome-azurophil granule fusion nor azurophil granule secretion (40). Accordingly, azurophil granule secretion appears to require not only the availability and transcellular movement of endosomes, but also an additional event(s) that controls the fusion between both organelles and that is regulated independently from endosome formation. Monensin, which seems to activate both endocytosis and fusion events in parallel through ionophore activity (7), should be a useful tool for identifying the control mechanisms involved.

\section{Acknowledgments}

We thank Ms. Sheryl Campbell and Ms. Lindsay Guthrie for their excellent technical assistance, and Ms. Brenda Sebern for skillfully preparing the manuscript.

This work was carried out in the F. L. Bryant, Jr., Research Laboratory for the Mechanisms of Lung Disease.

\section{References}

1. Bainton, D. F., and M. G. Farquhar. 1968. Differences in granule content of azurophil and specific granules of polymorphonuclear leukocytes. II. Cytochemistry and electron microscopy of bone marrow cells. J. Cell Biol. 39:299317.

2. Wetzel, B. K., S. S. Spicer, and R. G. Horn. 1967. Fine structural localization of acid and alkaline phosphatases in cells of rabbit blood and bone marrow. J. Histochem. Cytochem. 15:311-334. 
3. Henson, P. M. 1971. The immunologic release of constituents from neutrophil leukocytes. II. Mechanisms of release during phagocytosis, and adherence to nonphagocytosable surfaces. J. Immunol. 107:1547-1557.

4. Bentwood, B. J., and P. M. Henson. 1980. The sequential release of granule constitutents from human neutrophils. J. Immunol. 124:855-862.

5. Bainton, D. F. 1973. Sequential degranulation of the two types of polymorphonuclear leukocyte granules during phagocytosis of microorganisms. J. Cell Biol. 58:249-264

6. Wright, D. G., D. A. Bralove, and J. I. Gallin. 1977. The differential mobilization of human neutrophil granules. Effects of phorbol myristate acetate and ionophore A23187. Am. J. Pathol. 87:273-274.

7. Fittschen, C., and P. M. Henson. 1991. Selective secretion of azurophil granule contents induced by monovalent cation ionophores in human neutrophils: Evidence for direct ionophore effects on the granule membrane. J. Leuk. Biol. 50:517-528.

8. Volpi, M., P. H. Naccache, T. F. P. Molski, J. Shetcyk, C.-K. Huang, M. L. Marsh, J. Munoz, E. L. Becker, and R. I. Sha'afi. 1985. Pertussis toxin inhibits f-Met-Leu-Phe but not phorbol ester-stimulated changes in rabbit neutrophils: role of G-proteins in excitation-response coupling. Proc. Natl. Acad. Sci. USA. 82:2708-2712.

9. Wilson, E., W. G. Rice, J. M. Kinkade, Jr., A. H. Merrill, Jr., R. R. Arnold, and J. D. Lambeth. 1987. Protein kinase $C$ inhibition by sphingoid long-chain bases: effects on secretion in human neutrophils. Arch. Biochem. Biophys. 259:204-214.

10. Korchak, H. M., L. E. Rutherford, and G. Weissman. 1984. Stimulus response coupling in the human neutrophil. I. Kinetic analysis of changes in calcium permeability. J. Biol. Chem. 249:4070-4075.

11. Henson, P. M., and Z. G. Oades. 1973. Enhancement of immunologically induced granule exocytosis from neutrophils by cytochalasin B. J. Immunol. 110:290-293.

12. Zurier, R. B., S. Hoffstein, and G. Weismann. 1973. Cytochalasin B: effect of lysosomal enzyme release from human leukocytes. Proc. Natl. Acad. Sci. USA. 70:844-.

13. Smolen, J. E., and S. J. Stoehr. 1985. Micromolar concentrations of free calcium provoke secretion of lysozyme from human neutrophils permeabilized with saponin. J. Immunol. 134:1859-1865.

14. Philips, M. R., S. B. Abramson, S. L. Kolasinski, K. A. Haines, G. Weissmann, and M. G. Rosenfeld. 1991. Low molecular weight GTP-binding proteins in human neutrophil granule membranes. J. Biol. Chem. 266:1289-1298.

15. Barrowman, M. M., S. Cockcroft, and B. D. Gomperts. 1986. Two roles for guanine nucleotides in the stimulus-secretion sequence of neutrophils. Nature (Lond.). 319:504-507.

16. Henson, P. M. 1980. Mechanisms of exocytosis in phagocytic inflammatory cells. Parke-Davis Award Lecture. Am. J. Pathol. 101:494-511.

17. Hohman, T. C., and B. Bowers. 1984. Hydrolase secretion is a consequence of membrane recycling. J. Cell Biol. 98:246-252.

18. Haslett, C., L. A. Guthrie, M. M. Kopaniak, R. B. Johnston, Jr., and P. M. Henson. 1985. Modulation of multiple neutrophil functions by preparative methods or trace concentrations of bacterial lipopolysaccharide. Am. J. Pathol. 119:101-110.

19. Henson, P. M., B. Zanolari, N. A. Schwartzman, and S. R. Hong. 1978. Intracellular control of human neutrophil secretion. I. C5a-induced stimulus-specific desensitization and the effects of cytochalasin B. J. Immunol. 121:851-855.

20. Gottlieb, C., K. S. Lau, L. R. Wasserman, and V. Herbert. 1965. Rapid charcoal assay for intrinsic factor (IF), gastric juice unsaturated $\mathbf{B}_{12}$-binding capacity, antibody to IF, and serum unsaturated $\mathbf{B}_{12}$-binding capacity. Blood. 25:875-.

21. West, B. C., A. S. Rosenthal, N. A. Gelb, and H. R. Kimball. 1974. Separation and characterization of human neutrophil granules. Am. J. Pathol. $77: 41-66$
22. Riches, D. W. H., S. K. Young, J. F. Seccombe, J. E. Henson, K. L. Clay, and P. M. Henson. 1990. The subcellular distribution of platelet-activating factor in stimulated human neutrophils. J. Immunol. 145:3062-3070.

23. Graham, R. C., and M. J. Karnovsky. 1966. The early stages of absorption of injected horseradish peroxidase in the proximal tubules of mouse kidney. Ultrastructural cytochemistry by a new technique. J. Histochem. Cytochem. 14:291-302.

24. Korchak, H. M., B. A. Eisenstat, S. T. Hoffstein, P. B. Dunham, and G. Weissman. 1980. Anion channel blockers inhibit lysosomal enzyme secretion from human neutrophils without affectin generation of superoxide anion. Proc. Natl. Acad. Sci. USA. 77:2721-2725.

25. Parmley, R. T., J. M. Kinkade, Jr., D. T. Akin, C. S. Gilbert, and G. S. Guzman. 1988. Monensin disruption of neutrophil granule genesis. Am. J. Pathol. 133:537-548.

26. Besterman, J. M., and R. B. Low. 1983. Endocytosis: A review of mechanisms and plasma membrane dynamics. Biochem. J. 210:1-13.

27. Jesaitis, R. K., C. A. Dahinden, C. M. Chang, and A. J. Jesaitis. 1987. Investigations on the role of Golgi-mediated, ligand-receptor processing in the activation of granulocytes by chemoattractants: differential effects of monensin. Biochim. Biophys. Acta. 927:382-391.

28. Davis, B. H., E. McCabe, and M. Langweiler. 1986. Characterization of f-Met-Leu-Phe-stimulated fluid pinocytosis in human polymorphonuclear leukocytes by flow cytometry. Cytometry. 7:251-262.

29. Jesaitis, A. J., J. O. Tolley, R. G. Painter, L. A. Sklar, and C. G. Cochrane. 1985. Membrane-cytoskeletin interactions and the regulation of chemotactic peptide-induced activation of human granulocyte: the effects of dihydrocytochalasin B. J. Cell. Biochem. 27:241-253.

30. Davies, P., and A. C. Allison. 1978. Effects of cytochalasin B on endocytosis and exocytosis. Front. Biol. 46:143-160.

31. Bratton, D. L., E. Dreyer, J. M. Kailey, V. A. Fadok, K. L. Clay, and P. M. Henson. 1992. The mechanism of internalization of platelet-activating factor in activated human neutrophils. J. Immunol. 148:514-523.

32. Fuchs, R., S. Schmid, and I. Mellman. 1989. A possible role for $\mathrm{Na}^{+} / \mathrm{K}^{+}$-ATPase in regulating ATP-dependent endosome acidification. Proc. Natl. Acad. Sci. USA. 86:539-543.

33. Simchowitz, L., R. Ratzlaff, and P. DeWeer. 1986. Anion/anion exchange in human neutrophils. J. Gen. Physiol. 88:195-217.

34. Mellman, I., R. Fuchs, and A. Helenius. 1986. Acidification of the endocytic and exocytic pathways. Annu. Rev. Biochem. 55:663-700.

35. Ohkuma, S., Y. Moriyama, and T. Takano. 1982. Identification and characterization of a proton pump on lysosomes by fluorescein isothiocyanatedextran fluorescence. Proc. Natl. Acad. Sci. USA. 79:2758-2762.

36. Barasch, J., B. Kiss, A. Prince, L. Saiman, D. Gruenert, and Q. Al-Awqati. 1991. Defective acidification of intracellular organelles in cystic fibrosis. Nature (Lond.). 352:70-73.

37. Bradbury, N. A., T. Jilling, G. Berta, E. J. Sorscher, R. J. Bridges, and K. L. Kirk. 1992. Regulation of plasma membrane recycling by CFTR. Science (Wash. DC). 256:530-532.

38. Kemp, T., A. Schram-Doumont, R. Van Geffel, R. Kram, and C. Szpirer. 1986. Alteration of the N-Formyl-Methionyl-Leucyl-Phenylalaine-Induced response in cystic fibrosis neutrophils. Pediatr. Res. 20:520-526.

39. Henson, P. M. 1971b. The immunologic release of constituents from neutrophil leukocytes. I. The role of antibody and complement on nonphagocytosable surfaces or phagocytosable particles. J. Immunol. 107:1535-1546.

40. White, J. G., and R. D. Estensen. 1974. Selective labilization of specific granules in polymorphonuclear leukocytes by phorbol myristate acetate. $\mathrm{Am}$. J. Pathol. 75:45-60.

41. Henson, P. M., J. E. Henson, C. Fittschen, D. L. Bratton, and D. W. H Riches. 1992. Phagocytic cells: degranulation and secretion. In Inflammation: Basic Principles and Clinical Correlates. J. Gallin, I. Goldstein, and R. Snyderman, editors. Raven Press Ltd., New York. pp. 511-539. 